\title{
Wounds at risk of tetanus: an unexpected level of underimmunization in an Italian patient cohort
}

\author{
Gaia Bavestrello Piccini ( $\sim$ gaia.bavestrellopic01@universitadipavia.it) \\ UVC Brugmann - Site Victor Horta https://orcid.org/0000-0002-5618-7404 \\ Jean-Christophe Cavenaile \\ UVC Brugmann - Site Victor Horta \\ Maria Antonietta Bressan \\ Fondazione IRCCS Policlinico San Matteo
}

\section{Research article}

Keywords: Tetanus, immunization, prophylaxis, wounds, prevalence, vaccine, immunoglobulin, anamnesis

Posted Date: January 13th, 2021

DOI: https://doi.org/10.21203/rs.2.20169/v6

License: @) (i) This work is licensed under a Creative Commons Attribution 4.0 International License. Read Full License 


\section{Abstract}

Background: Tetanus is an acute and potentially fatal disease caused by Clostridium tetani, an extremely resilient pathogen. Approximately $5 \%$ of all visits to the Emergency Department are due to traumatic wounds and Clostridium tetani can infect these. According to several surveillance programs, the incidence of clinical tetanus in Italy is tenfold higher than in other industrialised countries. In 2010 , Italy accounted for 57 ( $77 \%$ ) of the 74 confirmed cases reported within the European Union. We decided to conduct a study to assess the proportion of patients immunised against tetanus in the Emergency Department (ED).

Methods: Data from patients who presented to the Emergency Department of the Fondazione IRRCS Policlinico San Matteo between April 2016 and November 2017 with wounds potentially at risk of infection with Clostridium tetani were analysed. Tetanus Quick Stick (TQS) was used to identify patients immunized again tetanus.

Results: Of the total of 1094 patients included, 506 had all the necessary data for analysis. Of these, 432 patients (85\%) had no protection against tetanus. When stratified for age, underimmunization was $92 \%$ in patients above 60 years, $81 \%$ in patients between 46 and 60 years, $80 \%$ in patients between 19 and 45 years and $73 \%$ in children up to 18 years. In patients above 60 years of age, men are less underimmunized than women (80 vs $96 \%, p=0.0095)$.

Conclusions: This study highlights a very low level of immunization against tetanus. This occurs at all age. It could explain the higher incidence of tetanus in Italy in comparison to other European countries.

The analysed data suggests the need of conducting awareness campaigns to increase the percent of patients vaccinated against Clostridium tetani.

\section{Background}

Tetanus is an acute and potentially fatal infectious disease caused by Clostridium tetani, a spore-forming gram-positive bacteria that is commonly found in the soil of warm and moist areas and may be carried in the intestinal tracts and faeces of humans and animals. These bacteria can contaminate humans through a cut, a puncture wound, a burn, or a scratch in the skin, which may even be superficial [1].

Traumatic wounds account for approximately $5 \%$ of all the visits to the Emergency Department, therefore the ED offers the first possibility to perform correct prophylaxis, when considering the risk of tetanus infection ${ }^{[2]}$.

Between 2001 and 2009, a total of 594 tetanus cases were notified in Italy, with an average annual incidence of 1.0/1,000,000 inhabitants. The mean annual number of reported deaths was 21. Moreover, the incidence of clinical tetanus in Italy has been shown to be tenfold higher than in other industrialised countries such as Canada and the United States, likely because of higher susceptibility levels ${ }^{[3]}$, since Italy is one of the countries with the highest percentage of the elderly population (aged more than 65 ) ${ }^{[4]}$. It has indeed been demonstrated that over $50 \%$ of individuals aged between 45 to 64 and over two-thirds of subjects aged 65 + have a level of tetanus antibodies lower than $0.01 \mathrm{IU} / \mathrm{ml}$ [3]

In 2010, Italy accounted for 57 (77\%) of the 74 confirmed cases reported in the EU and it has been continuously reporting the highest number of tetanus cases since 2006 , ranging between 53 and 64 cases per year ${ }^{[5,6]}$.

Although according to a recent analysis from the European Centre for Disease Prevention and Control there had been a decrease in the reported incidence between 2010 and $2014^{[7]}$, a further increase was observed in 2015, surveyed by the World Health Organization (WHO) ${ }^{[8]}$.

Such a trend is probably due to the introduction of the "universal" vaccination campaign for all infants which has led to an $86 \%$ reduction in incidence from the mid-1950s to the present days ${ }^{[9]}$. Nowadays, in Italy, tetanus affects only subjects who are either unvaccinated or inadequately vaccinated. The purpose of this study was to determine whether a specific category of patients at higher risk of not being vaccinated could be outlined.

This would allow to better direct preventive measures for the general population, in terms of prophylaxis, but also to know whether certain patients are more at risk of developing acute tetanus in the hospital setting.

\section{Methods}

\section{Ethics committee}

Ethical approval was granted by the Ethics Committee of the San Matteo Hospital and the study was approved by the Institutional Review Board. Patient's informed consent was not applicable seeing the retrospective analysis of this study.

\section{Study design}


We conducted a retrospective observational study to evaluate the prevalence of vaccination against tetanus in the patient population.

\section{Setting}

The study was conducted at the Fondazione IRCCS (Istituto di Ricovero e Cura a Carattere Scientifico) Policlinico San Matteo which is affiliated with the Faculty of Medicine of the University of Pavia (Italy). It is one of the largest teaching hospitals in Italy, hosting every year hundreds of medical students performing clinical rotations.

\section{Participants}

The study included patients presenting to the Emergency Department (ED) between April 2016 and November 2017, with wounds potentially at risk of tetanus infection.

\section{Study protocol}

Data on the consecutive ED visits in the aforementioned period were extracted from electronic patient records using a standard data collection form.

Patients have been subdivided according to their age into four categories: 0-18, 19-45, 46-60, 60+. Since the study was carried out between 2016 and 2017, the subdivision was done considering the age on the $31^{\text {st }}$ December 2016 . Age and nationality have not been considered as exclusion criteria but have been kept into consideration for the subsequent analysis of the results. Exclusion criteria were severely bleeding wounds in need of immediate surgical intervention, and inability to provide a reliable history (i.e. psychiatric disease, dementia or confusion, patient in traumatic shock, unconsciousness).

The type of wound was also recorded, to determine the main categories of wounds at higher risk of contamination with C. tetani.

A register of Microsoft Excel was used to collect the data for subsequent epidemiological and statistical analysis.

Comparisons between some of the proportions were performed using a two-sample test for equality of proportions with continuity correction.

\section{Tetanus Quick Stick}

The immune status of patients was determined through the use of the Tetanus Quick Stick ${ }^{\text {TM }}$ (TQS, Zentech, Angleur, Belgium), a point of care testing which allows to conclude whether patients dispose of protecting levels of circulating antibodies against tetanus.

The TQS is an immunochromatographic test that utilises a combination of tetanus toxoid coated on the solid phase together with a tetanus toxoid dye conjugate for the rapid detection of anti-tetanus antibodies in human serum, plasma, or whole blood ${ }^{[10]}$. It is indicated for:

- the determination of the real immune status,

- the identification of unprotected individuals,

- prevention of side reaction due to unnecessary vaccination,

- the follow-up of vaccinated immunodeficient patients.

The TQS has been evaluated in several ED worldwide and it has been compared with the gold standard enzyme-linked immunosorbent assay (ELISA). Its sensitivity and specificity have been estimated to be 76 to $88 \%$ and 97 to $100 \%$ respectively ${ }^{[11,12,13]}$, therefore it can be considered as a reliable tool for screening patients that do not need to receive anti-tetanus prophylaxis ${ }^{[14]}$. There are no contraindications to this test ${ }^{[15]}$.

\section{Statistics}

Standard statistical analyses were performed, including mean, standard deviation, and ANOVA for repeated measures to test the between- and within-subject effect after Kolmogorov-Smirnov testing for normality. The statistical analysis of the data has been performed through the Welch two-sample t-test. All statistical analyses were performed with the GraphPad Prism version 5.00 for Windows (GraphPad Software, La Jolla, CA, USA).

\section{Results}

On a total of 1094 patients included in our study 474 patients had incomplete data leaving 620 patients but 114 had no TQS so finally 506 patients were included in our study. Three quarters of the patients were males. Most patients were Italians (477) and 29 were foreigners. 
The results showed that 432 (85\%) patients out of 506 were unprotected against tetanus infection.

When stratified for age, underimmunization was $92 \%$ in patients above 60 years, $81 \%$ in patients between 46 and 60 years, $80 \%$ in patients between 19 and 45 years and $73 \%$ in children up to 18 years. Underimmunization was significantly higher $(92$ vs $80 \%$, p <0.0001) respectively in patients above 60 years than those under 60 years. Moreover, among patients above 60 years, underimmunization was significantly higher in women than in men ( 96 vs $80 \%, p=0.0095)$ respectively.

From the data analysis, when taking into consideration the number of patients for every age range who presented with wounds to the ED (Figure 3 ), it is possible to see that even younger patients lack protective immunity (Figure 1, Table 1).

Table 1: Percentage of unprotected patients, according to age

\begin{tabular}{|l|c|c|}
\hline $\begin{array}{c}\text { Age } \\
\text { (years) }\end{array}$ & $\begin{array}{c}\text { Total patients } \\
(\mathrm{n})\end{array}$ & $\begin{array}{c}\text { Non-immunized patients } \\
(\mathrm{n}, \%)\end{array}$ \\
\hline$>60$ & 238 & $219(92)$ \\
\hline $46-60$ & 125 & $100(81)$ \\
\hline $19-45$ & 128 & $102(80)$ \\
\hline $0-18$ & 15 & $11(73)$ \\
\hline
\end{tabular}

\section{Discussion}

Our study has demonstrated that $85 \%$ of the medical files showed no protection against tetanus infection. Underimmunization changes with age groups.

It is essential to focus on the fact that vaccination against tetanus in Italy is currently mandatory at the age of 3 months, 5 months, 11 months, and 6 years in individuals born after 2001. A second booster dose is also mandatory at 12-18 years for them. Afterwards, TDAP (Tetanus Diphtheria and Pertussis) is recommended every 10 years from age 19, and for pregnant women in the third trimester (ideally 28 weeks) ${ }^{[16,17]}$. It is therefore surprising that even the subset of patients aged 0 to 18 years has been found to be unprotected (73\%) against tetanus infection.

The lower rates of protection present even among the younger patients might be due to factors such as a lack of knowledge about the importance of prevention of this disease through a complete cycle of vaccinations, as well as a lack of awareness on the necessity to receive boosters once completed the primary immunization series. This factor is probably a consequence of the fact that tetanus is currently one of the most underestimated and less well-known possible complications of a wound.

As reported by other authors, the most relevant factor associated with a lower immunity rate was found to be increased age ${ }^{[3,18,19]}$. This finding is possibly due to a combination of the lack of systematic vaccination before 1962,an increased life expectancy and the lack of administration of the recommended tetanus booster, the decline of tetanus protective antibody levels as age increases, and a deficient immune response to the vaccine which is associated with immunosenescence $[15,18,20,21,22,23]$.

These data confirm the fact that the elderly population is more at risk since they are less covered by the vaccine. We can therefore say that the analysed group of patients is representative of the Italian reality as described by the Italian Ministry of Health [19]. The lack of immunity can also directly result in an increased incidence of tetanus in elderly patients ${ }^{[24]}$.

In patients older than 60 years, there is a difference in immunization rates between men and women (Figure 2). A slightly higher percentage of men (20\%) were protected against tetanus as compared to women (4\%). The fact that the Military Service is not obligatory anymore since 2005 ${ }^{[25]}$, but also to the introduction of the obligatory vaccination schedule in $1968^{[26]}$, and to the more recent law ${ }^{[27,28]}$, which reaffirms that for the individuals of age comprised between 0-16 years old, a series of vaccinations have to be rendered mandatory and administered without charge. These elements could induce, during the next years, a possible increase in patients immunized against tetanus. This could also decrease the sexinduced difference in immunization.

Another important issue when considering high-risk groups populations is the one of immigration. Even though Pavia is not as cosmopolitan as other cities that have been taken into account by other studies, such as Rome and Brussels, the recent increase in migrants from other countries in which the healthcare system is not so developed, could be responsible for the possible decrease in vaccination coverage over the next years. In this study, it was not possible to underline major discrepancies concerning the difference in immune coverage among patients of different nationalities, due to the small sample of foreign patients (34). However, this investigation could be an interesting topic on which to conduct subsequent researches. 
In order to increase the prevalence of immunization among patients, prevention should be done, as suggested by the Ministerial Circular concerning the recent Decree Law ${ }^{[29]}$, by promoting vaccinations both for new-borns, and older patients who somehow did not complete the primary vaccination schedule. Better compliance with vaccine coverage has also been demonstrated to be associated with fewer hospital admissions in children ${ }^{[30]}$.

Prevention should be done both through notifications under the form of letters, emails, leaflets, but also in a more direct way when the patient presents to the hospital or the cabinet of the general practitioner.

From a clinical point of view, this study underlines the importance of considering all individuals presenting to the Emergency Department with wounds at risk of contracting a tetanus infection. It is indeed demonstrated that most individuals in Italy, despite their age and gender, could be at risk of being not immunized against tetanus infection. It would be therefore important to either consult the patients' vaccination card or, to assess their level of antibodies against tetanus through a rapid diagnostic test for the patients without a definite proof of a completed vaccination series.

The use of a rapid test would allow the detection of non-protected individuals, and it would help to choose the correct prophylaxis that has to be administered in the Emergency Department. Such a protocol, in the long run, would also help reduce the number of patients who are not protected against tetanus infection.

Patients should be made aware of the importance of keeping track of their immunization status, and of remembering the date of the last administration of the vaccine, especially in regions where rapid diagnostic tests are not available.

Moreover, it would be extremely important to more comprehensively educate patients on the topic of vaccines and vaccine-preventable infections, stressing on the fact that some of these infections could quickly lead to significant complications, and eventually result in the death of the individual.

\section{Conclusion}

Our study confirmed one of Italy's town exceptionally low percentage of immunisation against tetanus. Indeed $85 \%$ of our medical files showed no protection against tetanus infection. Immunization changes with age groups, younger patients being less underimmunized than older patients. In patients above 60 years old, men seem less underimmunized than women. The introduction of the obligatory vaccination schedule in 1968 , and the more recent law reaffirming that for the individuals of age comprised between 0-16 years old, a series of vaccinations have to be rendered mandatory and administered without charge. These elements could induce, during the next years, a possible increase in patients immunized against tetanus. This could also decrease the sex-induced difference in immunization.

\section{Abbreviations}

C. tetani: Clostridium tetani

ED: Emergency Department

TQS: Tetanus Quick Stick

POCT: Point Of Care Testing

ELISA: Enzyme-Linked Immunosorbent Assay

WHO: World Health Organization

IRCCS: Istituto di Ricovero e Cura a Carattere Scientifico

EU: European Union

\section{Declarations}

- Ethics approval and consent to participate: Informed consent of the patients was deemed not necessary because of the retrospective design of the study, and exemption of ethical approval was granted by the Ethics Committee of the San Matteo Hospital. The study was approved by the Institutional Review Board.

- Consent for publication: Not applicable

- Availability of data and material: The datasets used and/or analysed during the current study are available from the corresponding author on reasonable request.

- Competing interests: The authors declare that they have no competing interests 
- Funding: Not applicable

- Authors' contributions: GBP: Designed the study, analysed, and interpreted data. JCC: Designed and supervised the study, edited multiple manuscript drafts. MAB: collected data. All authors have read and approved the manuscript.

- Acknowledgements: Giovanni Ricevuti, Stefano Perlini, Gabriele Savioli

- Authors' information (optional): Not applicable

\section{References}

1. Pellizzari R, Rossetto O, Schiavo G, Montecucco C. Tetanus and botulinum neurotoxins: mechanism of action and therapeutic uses. Philos Trans R Soc Lond B Biol Sci. 1999 Feb 28; 354(1381): 259-268.

2. Prevaldi C, Paolillo C, Locatelli C, et al. Management of traumatic wounds in the Emergency Department: position paper from the Academy of Emergency Medicine and Care (AcEMC) and the World Society of Emergency Surgery (WSES). World J Emerg Surg. 2016;11:30. Published 2016 Jun 18. doi:10.1186/s13017-016-0084-3

3. Filia A, Bella A, von Hunolstein C, et al. Tetanus in Italy 2001-2010: a continuing threat in older adults. Vaccine. 2014;32:639-644.

4. https://data.worldbank.org/indicator/SP.POP.65UP.TO.ZS?end=2018\&most_recent_value_desc=true\&start=2018\&view=map\&year=2018. Accessed 24 Mar 2020

5. European Centre for Disease Prevention and Control. Annual Epidemiological Report 2012. Reporting on 2010 surveillance data and 2011 epidemic intelligence data. Stockholm: ECDC; 2013.

6. http://www.ansa.it/canale_saluteebenessere/notizie/sanita/2017/06/28/vaccini-bambino-senza-copertura-ricoverato-per-tetano_1 edb20895bc6-43d1-b522-0b922c03255d.html. Accessed 05 Sep 2017

7. European Centre for Disease Prevention and Control. Annual Epidemiological Report 2016 - Tetanus. Stockholm: ECDC; 2016.

8. WHO/IVB database, data reported to WHO by Member States. http://apps.who.int/ immunization_monitoring/globalsummary/timeseries/tsincidencediphteria.html. Accessed 05 Sep 2017

9. Mandolini D, Ciofi degli Atti M, Pedalino B, Bella A, De Mei B et al. Epidemiology of tetanus in Italy Bollettino Epidemiologico Nazionale; 2002 (3)

10. Information leaflet of the TQS produced by ZenTech. http:// bionicum.com.pl/pliki/pdf/TQS-S-GZ-VZB-001-rev0.pdf. Accessed 20 July 2017

11. Colombet I, Saguez C, Sanson-Le Pors MJ, Coudert B, Chatellier G, Espinoza P: Diagnosis of tetanus immunization status: multicenter assessment of a rapid biological test. Clin Diagn Lab Immunol 2005;12(9):1057-1062

12. Hatamabadi HR, Abdalvand A, Safari S, Kariman H, Dolatabadi AA, et al.Tetanus quick stick as an applicable and cost-effective test in assessment of immunity status. Am J Emerg Med 2011, 29(7):717-720.

13. Paulke-Korinek M, Rendi-Wagner P, Kundi M, Tomann B, Wiedermann U, Kollaritsch H. Pretravel consultation: rapid dipstick test as a decision guidance for the application of tetanus booster vaccinations. Journal of travel medicine 2008;15(6):437-441 Doi: 10.1016/j.it. 2009.05.002 PMC. Web. 29 Sept.2017

14. Golikhatir I, Montazer SH, Bagheri N, et al. Diagnostic Accuracy of Quick Stick for Identifying Traumatic Patients in Need of Tetanus Prophylaxis; a Cross-sectional Study. Emergency 2017;5(1):e66

15. Orsi G, Modini C, Principe M, Di Muzio M, Moriconi A, Amato M, et al. Assessment of tetanus immunity status by tetanus quick stick and anamnesis: a prospective double blind study. Ann Ig. 2015;27(2):467-474.

16. http://www.salute.gov.it/imgs/C_17_pubblicazioni_2571_allegato.pdf. Accessed 24 Mar 2020

17. https://vaccine-schedule.ecdc.europa.eu/Scheduler/ByCountry? SelectedCountryld=103\&IncludeChildAgeGroup=true\&IncludeChildAgeGroup=false\&IncludeAdultAgeGroup=true\&IncludeAdultAgeGroup=false . Accessed 24 Mar 2020

18. Hainz U, Jeneweih B, Asch E, Pfeiffer KP, Berger P, Grubeck-Loebenstein B. Insufficient protection for healthy elderly adults by tetanus and TBE vaccines. Vaccine 2005; 23(25):3232-3235.

19. Italian Ministry of Health. Decree N. 16 of 11 November 1996. Tetanus prophylaxis measures. (Circolare n.16 del 11/11/1996. Tetano: misure di profilassi).

20. Chen WH, Kozlovsky BF, Effros RB, Grubeck-Loebenstein B, Edelman R, Sztein MB. Vaccination in the elderly: an immunological perspective. Trends in immunology 2009;30(7): 351-359.

21. Michel JP, M. Gusmano, Blank PR, Philp I, Vaccination and healthy ageing: How to make life-course vaccination a successful public health strategy. European Geriatric Medicine 2010;1:155-165.

22. Targonski PV, Jacobson RM, Poland GA. Immunosenescence: role and measurement in influenza vaccine response among the elderly. Vaccine 2007;25:3066-3069

23. Pawelec G, Akbar A, Caruso C, et al. Human immunosenescence: is it infectious? Immunol Rev 2005;205:257-268 
24. Centers for Disease Control. Tetanus Surveillance-United States, 1995-1997. MMWR 1998;47:1-13.

25. Legge n. 226 del 23 agosto 2004, in G.U. n. 204 del 31 agosto 2004

26. Legge n. 166 del 27 aprile 1981, in G.U. n. 119 del 2 maggio 1981

27. Decreto legge n. 73 del 7 giugno 2017, in G.U. n. 130 del 7 giugno 2017

28. Legge n. 119 del 31 luglio 2017, in G.U. n. 182 del 5 agosto 2017

29. Italian Ministry of Health. Newsletter 16/08/2017. Urgent instructions regarding prevention through vaccines, infectious diseases and controversies in the administration of drugs (Circolare del 16/08/2017. Disposizioni urgenti in materia di prevenzione vaccinale, di malattie infettive e di controversie relative alla somministrazione di farmaci)

30. Cecil, E., Bottle, A., Ma, R. et al. Impact of preventive primary care on children's unplanned hospital admissions: a population-based birth cohort study of UK children 2000-2013. BMC Med 16, 151 (2018) doi:10.1186/s12916-018-1142-3

\section{Figures}

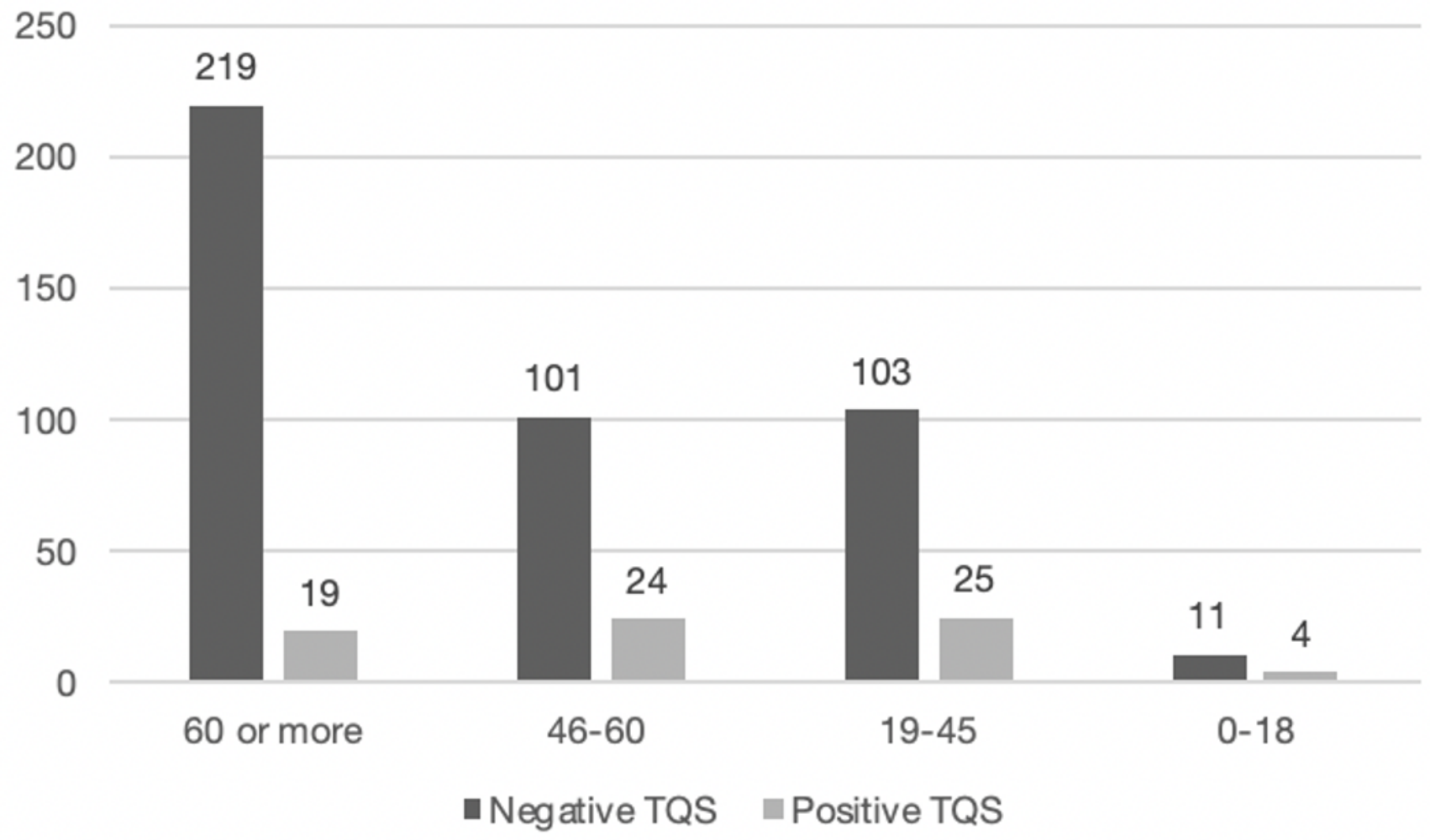

\section{Figure 1}

TQS results with regards to age class of patients 


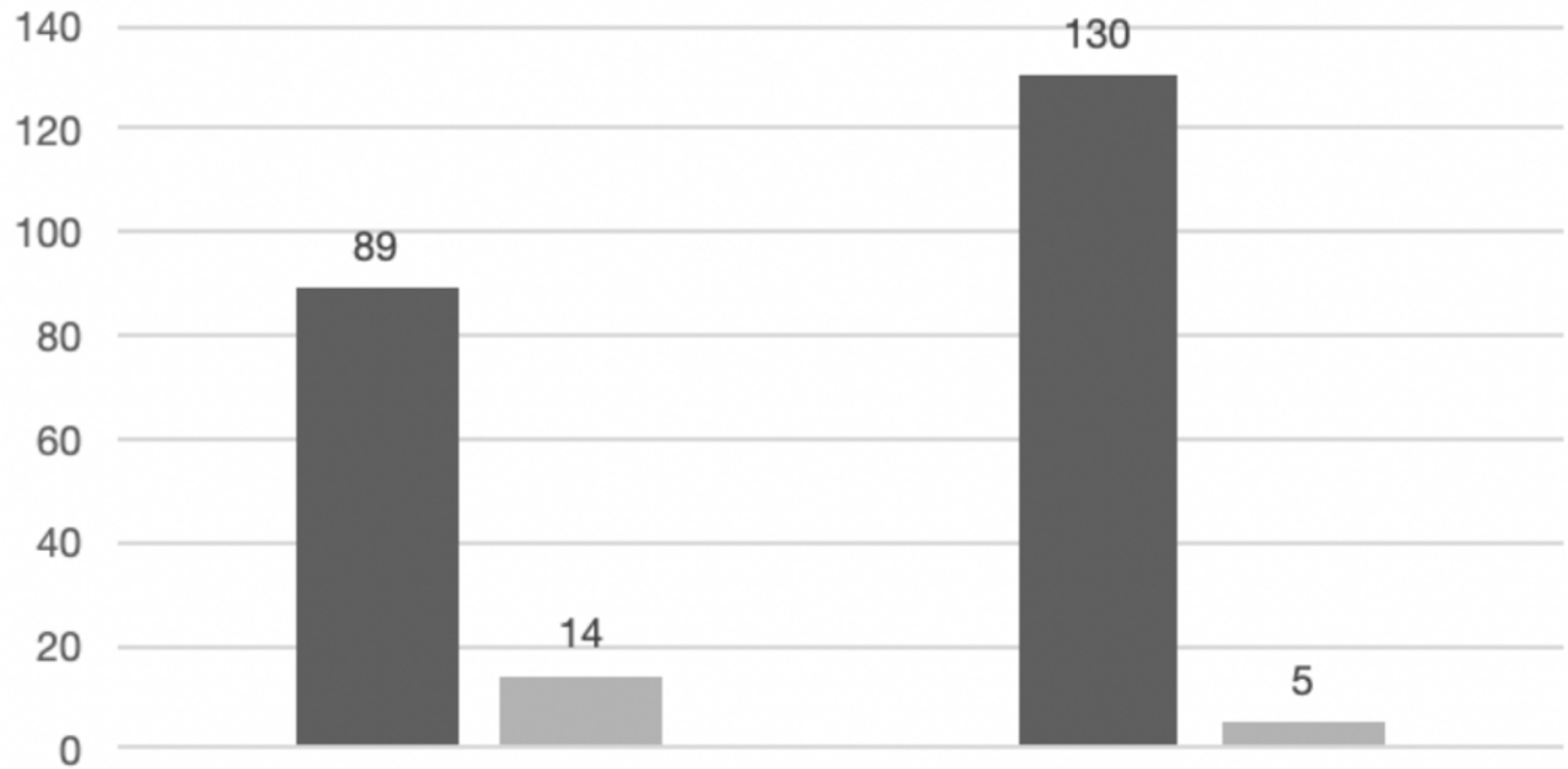

Men

Female

- Negative Mositive

Figure 2

TQS results according to gender in patients aged $>60$

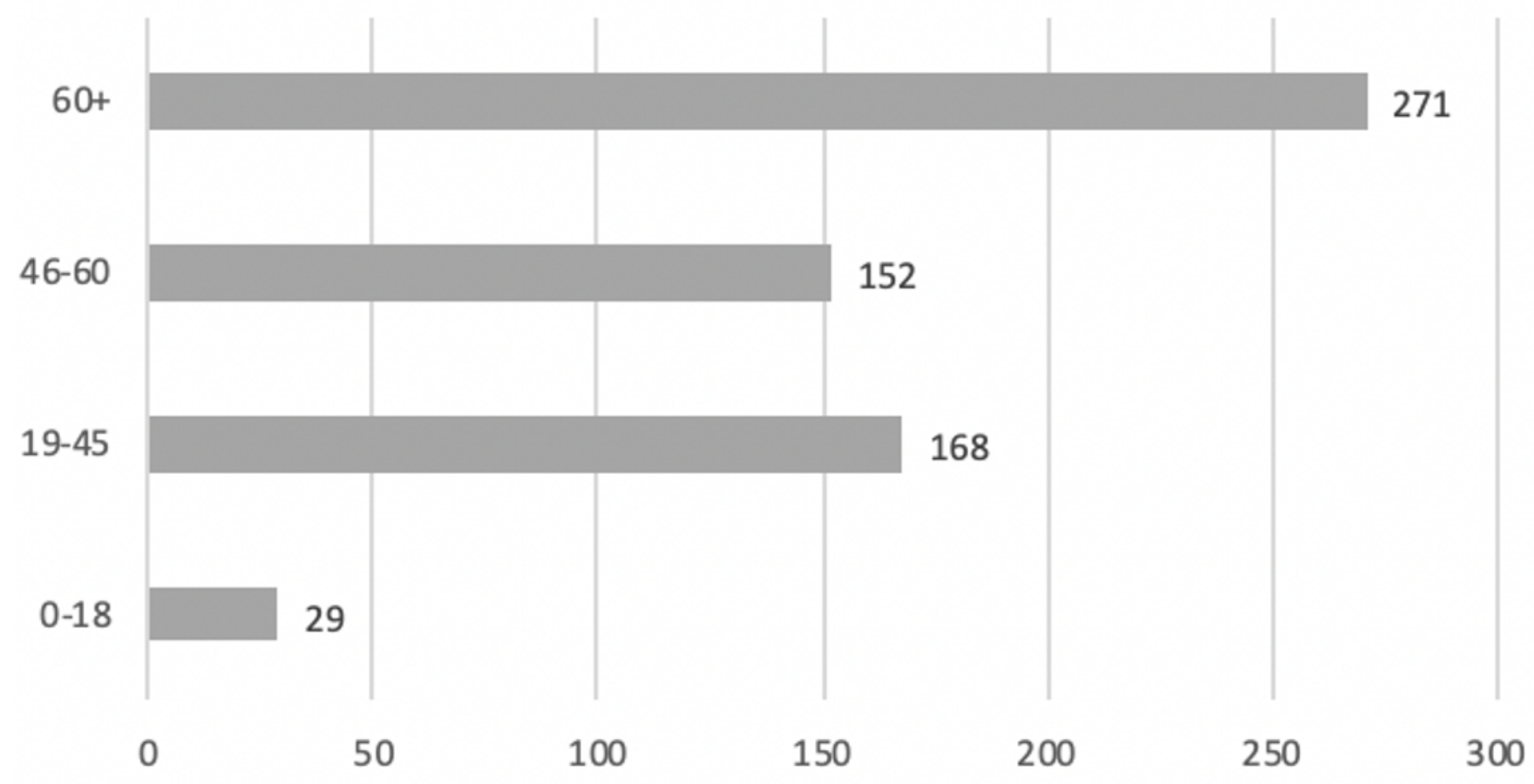

Figure 3

Subdivision of patients accessing the Emergency Department for wounds in age classes 